\title{
Simulación numérica del agua subterránea en el acuífero Guadalupe, Ensenada, Baja California, México: Caso de estudio, condiciones estacionarias y transitorias
}

\author{
Numerical simulation of the groundwater in the Guadalupe aquifer, Ensenada, \\ Baja California, Mexico: Case study in steady- and transient-state conditions \\ Alejandro Figueroa-Núñez*, José Rubén Campos-Gaytán*® \\ Figueroa-Núñez, A., \& Campos-Gaytán, J. R. (2018). Simulación numérica del agua \\ subterránea en el acuífero Guadalupe, Ensenada, Baja California, México: Caso \\ de estudio, condiciones estacionarias y transitorias. Investigación y Ciencia de la \\ Universidad Autónoma de Aguascalientes, 26(75), 46-54.
}

RESUMEN

La ciudad de Ensenada en Baja California, México, presenta una problemática de agua potable, cuyo suministro es en su mayoría de fuentes subterráneas. El acuífero Guadalupe es una de las principales fuentes de abastecimiento para la ciudad y la región productora de vino más importante del país, el Valle de Guadalupe. Se presenta un modelo de simulación de agua subterránea en condiciones estacionarias y transitorias utilizando MODFLOW-2005 y tomando como base un modelo previo. Con base en los resultados de la simulación, la comparación entre los niveles freáticos simulados y observados en la fecha definida como condición estacionaria (febrero de 1984) y transitoria (19842008), la conclusión es que el ajuste presentado es aceptable y que el comportamiento del flujo hidráulico se reproduce satisfactoriamente; con un RMSE de $3.25 \mathrm{~m}$ para el estado estacionario y de $8.36 \mathrm{~m}$ para el transitorio.

Palabras clave: acuíferos; modelo de simulación; agua subterránea; MODFLOW; Valle de Guadalupe; Baja California.

Keywords: aquifer; groundwater; simulation model; MODFLOW; Guadalupe Valley; Baja California.

Recibido: 17 de enero de 2018, aceptado: 30 de julio de 2018

* Facultad de Ingeniería, Arquitectura y Diseño, Universidad Autónoma de Baja California. Carretera Transpeninsular Ensenada-Tijuana \# 3917 Colonia Playitas, C. P. 22860, Ensenada, Baja California, México. Correo electrónico: alejandro.figueroa@uabc.edu.mx; rcampos@uabc.edu.mx

Autor para correspondencia
ABSTRACT

The city of Ensenada, Baja California, Mexico, presents a problem with the water supply, which is mostly from underground sources. The Guadalupe aquifer is one of the main sources of water supply for the city and for the most important wineproducing region in the country, the Guadalupe Valley. This article presents a groundwater simulation model in steady- and transient-state conditions, which used MODFLOW-2005 and taking as base a previous model. Based on the simulation results, the comparison between the simulated and observed hydraulic heads for the date defined as a steadystate- (February 1984) and transient-state conditions (1984-2008), this model has an acceptable vertical match, and the behavior of the hydraulic flow is achieved satisfactorily. In addition, a RMSE of $3.25 \mathrm{~m}$ for steady state and $8.36 \mathrm{~m}$ for transient conditions were obtained.

INTRODUCCIÓN

Conocer el comportamiento del agua subterránea y las características físicas de un acuífero permite un mejor manejo para cualquier comunidad. La toma de decisiones con respecto a tasas de extracción se puede facilitar y optimizar si se conoce la manera en que las mismas afectarán los niveles freáticos en las fuentes de abastecimiento subterráneas. Para lograr lo anterior, los modelos de simulación pueden ser utilizados para entender el comportamiento de los acuíferos y con esto evaluar distintas estrategias de manejo para los mismos (Dentoni, Deidda, Paniconi, Qahman, \& Lecca, 2015). 


\section{IIVESTIGAGIÓn Y CUERCIA DE LA UNIVERSIDAD AUTÓnOMF DE RGUASCALIERTES}

Los modelos de simulación del flujo de agua subterránea se han desarrollado ampliamente por diversos autores alrededor del mundo, quienes han utilizado técnicas y herramientas computacionales como MODFLOW-2005 (Harbaugh, 2005) y SPRING (König et al., 2017) para representar el comportamiento de un acuífero, bajo condiciones y características específicas de cada zona.

En este artículo se presenta un modelo de simulación del flujo de agua subterránea en los estados estacionario y transitorio del acuífero Guadalupe (AG) ubicado en Baja California, México, mediante MODFLOW-2005 (Harbaugh, 2005). La finalidad es contar con un modelo actualizado del $A G$, que al mismo tiempo permita la compatibilidad con otros programas de computadora que solucionen problemáticas de optimización de acuíferos o utilidades agrícolas, por mencionar algunas. Un ejemplo podría ser el uso conjunto con el software Groundwater Management Process (GWM) (Ahlfeld, Baker, \& Barlow, 2009), una aplicación para el desarrollo de modelos de optimización de agua subterránea para establecer las tazas de extracción o recarga de pozos, límites máximos o mínimos a los que puede descender el nivel freático, entre otras variables, con la intención de establecer un programa de manejo del acuífero.

El AG es una de las principales fuentes de abastecimiento de agua potable para la ciudad de Ensenada y al mismo tiempo de la región productora de vino más importante del país, el Valle de Guadalupe (figura 1). En el estado de Baja California se produce $90 \%$ del vino elaborado en México y el Valle de Guadalupe representa la zona más emblemática del estado (Camacho Garza, 2016). El AG ha manifestado problemas de sobreexplotación (SEMARNAT, 2013), por lo que el presente trabajo representa una alternativa de solución de dicha problemática.

La ciudad de Ensenada, Baja California, México, presenta una problemática con el abasto de agua potable y, en el último año, $79 \%$ se suministró de fuentes subterráneas (Comisión Estatal del Agua [CEA], s. f.). Entre las principales causas se encuentran falta de lluvia, intrusión salina, falta de reutilización del agua residual tratada y sobreexplotación de los acuíferos. Esta última es una de las más importantes para el AG, ya que se tiene in situ una sobreexplotación de $12.04 \mathrm{Mm}^{3}$ /año (SEMARNAT, 2013) que ha reducido el volumen de agua disponible en el acuífero.

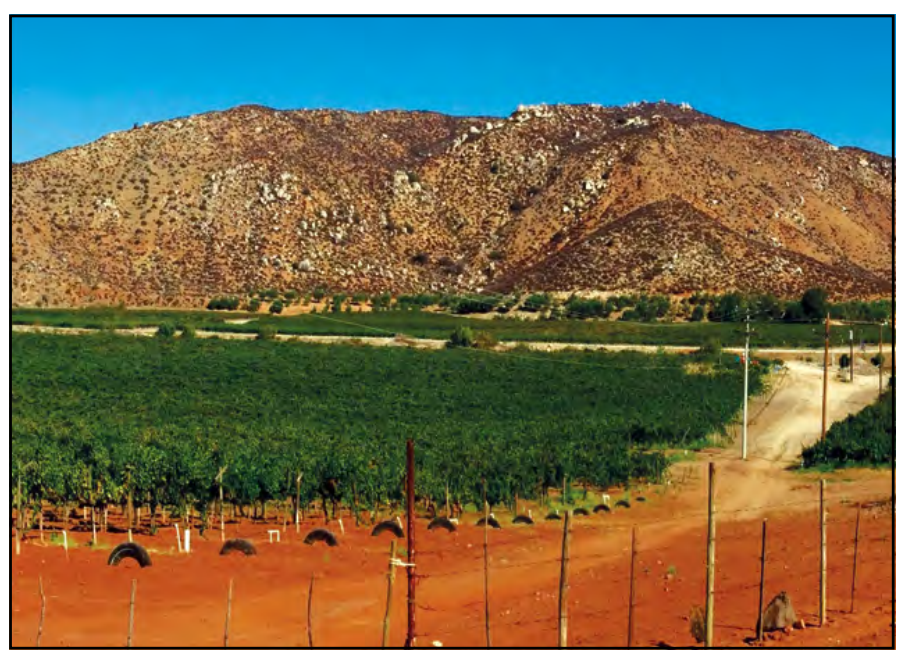

Figura 1. Vista típica de la agricultura en el Valle de Guadalupe. Elaboración propia.

Últimamente la reducción del volumen de agua en el AG para satisfacer la demanda se ha hecho obvia para la ciudad de Ensenada como consecuencia de la disminución de extracciones que la Comisión Estatal de Servicios Públicos de Ensenada (CESPE) realiza de los pozos que se ubican en el acuífero, las cuales representaban $25 \%$ del total de la demanda en 2011 (CEA, s. f.), mientras que en 2017 ya no se extrae agua del acuífero para suministro de la ciudad (CEA, s .f.). Los acuíferos que abastecen a la ciudad son Guadalupe, La Misión, Ensenada y Maneadero, este último presenta también intrusión salina.

En el AG se han realizado estudios similares al presente, como el de Campos-Gaytán y Kretzschmar (2006), quienes desarrollaron un modelo numérico para la simulación del agua subterránea en el AG en estado estacionario. Campos-Gaytán, Kretzschmar y Herrera-Oliva (2014), con base en lo publicado por Campos-Gaytán y Kretzschmar (2006), publicaron un modelo modificado con respecto a la descarga de agua del acuífero, principalmente cambiando pérdidas a través de fallas y fracturas por evapotranspiración y drenes, con el objetivo de mejorar el ajuste entre los niveles freáticos observado en campo y simulado. Realizaron simulaciones numéricas considerando tres escenarios de manejo del AG. En el primero consideraron una precipitación promedio reducida y una extracción promedio; en el segundo condiciones climáticas de recarga y descarga promedio; mientras que en el tercero una precipitación promedio reducida y extracciones que generarían una condición sustentable del acuífero. 
IIVESTIGACIÓn Y CIERCIA DE LA UחIVERSIDAD AUTÓNOTH

48

El modelo presentado en este artículo se basa en el de Campos-Gaytán et al. (2014) e incluye modificaciones destacables: el software utilizado es MODFLOW-2005 (Harbaugh, 2005); la topografía superficial se introduce mediante un modelo digital de elevaciones (MDE) del estado de Baja California, publicado por el INEGI (s.f.); la discretización es en celdas de mejor resolución (i.e., menor tamaño); se encuentra georreferenciado; la manera en que se recarga el acuífero, entre otras.

\section{MATERIALES Y MÉTODOS}

\section{Zona de estudio}

Localizada en el noroeste de México, la cuenca Guadalupe tiene una superficie de aproximadamente $2,400 \mathrm{~km}^{2}$, se ubica entre los paralelos $31^{\circ} 51^{\prime}$ y $32^{\circ} 15^{\prime}$ norte y los meridianos $115^{\circ} 52^{\prime}$ y $116^{\circ} 51^{\prime}$ oeste; se puede subdividir para su estudio en las subcuencas Ojos Negros, Guadalupe y La Misión (Campos-Gaytán, 2008; CONAGUA, 2015) (figuras 2 y 3). La zona de estudio es el acuífero que subyace la subcuenca del Valle de Guadalupe, misma que presenta clima mediterráneo semiárido con temperatura promedio anual de $15.7^{\circ} \mathrm{C}$ (Villarreal Rodríguez, 2012) y precipitaciones en invierno (diciembre-marzo). Con base en la información de Campos-Gaytán (2008), Herrera-Oliva, Campos-Gaytán y Carrillo-González (2017) y de la Secretaría de Fomento Agropecuario del Estado de Baja California (SEFOA, s. f.), la precipitación media anual de la zona de estudio es de 342 mm/año (1977-2016) (figura 4).

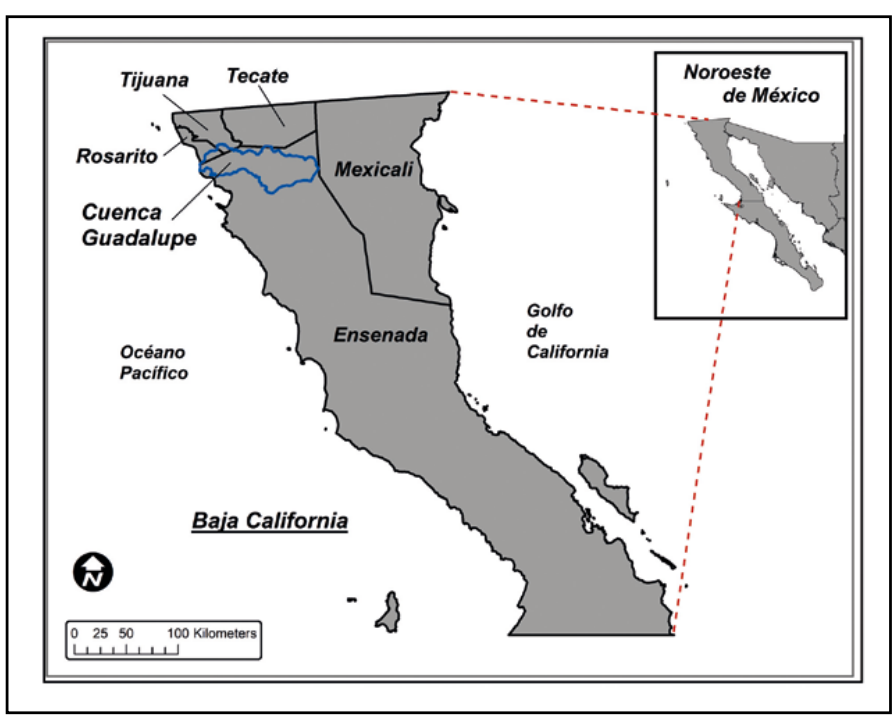

Figura 2. Localización de la zona de estudio. Límites del estado de Baja California y sus municipios (CONABIO, 2004).

Elaboración propia.

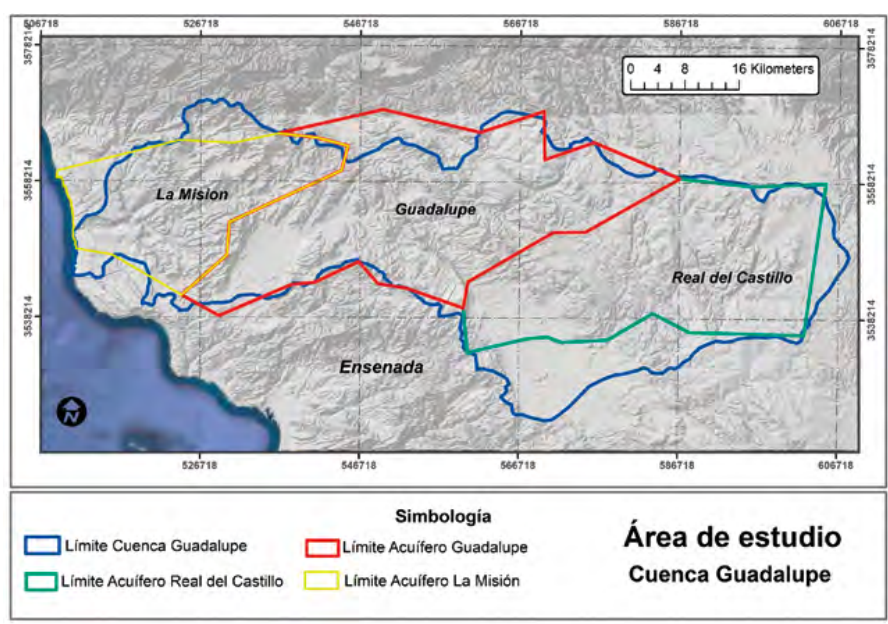

Figura 3. Distribución administrativa de la cuenca Guadalupe y los acuíferos que la componen.

Elaboración propia.

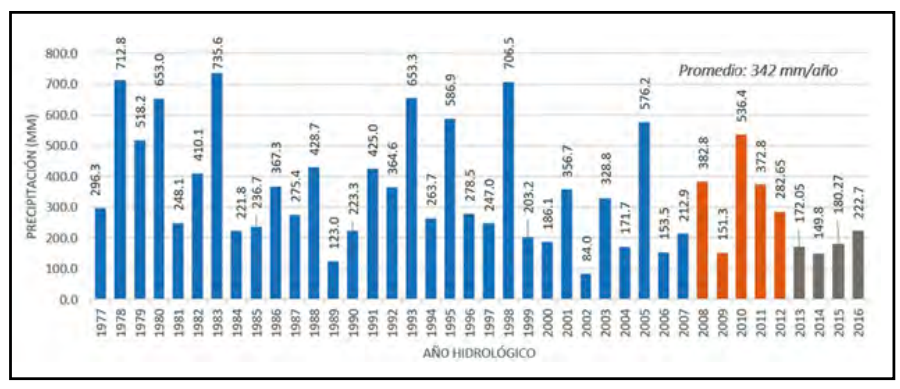

Figura 4. Precipitación media anual en mm durante el periodo 1977-2016. Información para 1977-2007 de Campos-Gaytán (2008), 2008-2012 de Herrera-Oliva et al. (2017) y 2013-2016 de la SEFOA (s. f.).

Elaboración propia.

\section{Hidrogeología}

En el Valle de Guadalupe se pueden encontrar rocas ígneas intrusivas y extrusivas, rocas metamórficas y depósitos aluviales (figura 5). Las rocas ígneas intrusivas del Cretácico son las que más afloran en la zona de estudio, están constituidas en orden de abundancia por granodioritas, granitos, dioritas y gabros. Las ígneas extrusivas del Mioceno afloran principalmente hacia el oeste de la fosa El Porvenir. Estas rocas están constituidas porbasaltos y andesitas, principalmente. Las metamórficas del Paleozoico, con menor abundancia de afloramiento, están expuestas hacia el este de la fosa El Porvenir, y se encuentran representadas por esquistos y pizarras.

Hacia la parte suroeste de la fosa El Porvenir y hacia el este de la fosa Calafia algunos cerros y lomeríos muestran la existencia de conglomerados acomodados como abanicos aluviales. Los depósitos aluviales integran el AG en todo lo largo del 


\section{IIVESTIGAGIÓn Y CUERCIA DE LA UNIVERSIDAD AUTÓnOMF DE RGUASCALIERTES}

Arroyo Guadalupe y están formados por materiales granulares no consolidados medianamente clasificados. Al menos tres sistemas de fallamiento pueden distinguirse en la zona de estudio; el primero está compuesto por varias fallas normales localizadas al oeste de la zona de estudio, como ejemplo las fallas El Porvenir y Guadalupe. Dentro del segundo sistema se puede señalar la falla Calafia, que también es una falla normal, localizada al este de la fosa Calafia. En el tercer sistema de fallamiento se encuentra la falla El Barbón, localizada en la porción noreste del valle y corresponde al tipo normal (Campos-Gaytán, 2008).

Con base en la información geológica, Campos-Gaytán (2008) y Campos-Gaytán et al. (2014) delimitaron la zona activa de su modelo diferenciando al área permeable de la impermeable. La reducción del tamaño de las celdas utilizadas en el modelo aquí presentado (de $500 \mathrm{~m} \times 500 \mathrm{~m}$ a $100 \mathrm{~m}$ x $100 \mathrm{~m}$ ) permitió realizar un modelo más apegado a la geología, ampliando la zona permeable (dominio de flujo del modelo) (figura 5).

El AG se puede dividir en dos zonas para su estudio: la fosa Calafia situada al noreste del valle y la fosa El Porvenir al sureste. El acuífero está compuesto por gravas, arenas, limos y arcillas poco consolidadas, permite el flujo de agua a través de ellas y la recarga del AG en grandes cantidades (Campos-Gaytán, 2008), cuyas conductividades hidráulicas varían desde 41.09 hasta $109.59 \mathrm{~m} /$ día (figura 6); mientras que las rocas ígnea y metamórfica que integran el área inactiva del modelo y establecen el límite del acuífero se consideran con una conductividad hidráulica de $0.0 \times 10^{\circ} \mathrm{m} /$ día.

\section{Descripción del modelo de simulación del AG}

Para simular el flujo de agua subterránea en el AG se empleó el MODFLOW-2005 (Harbaugh, 2005) mediante la interfaz ModelMuse (Winston, 2009) y se utilizó como base el modelo de flujo de agua subterránea reportado por Campos-Gaytán et al. (2014). Ellos describen el modelo conceptual del AG como un acuífero libre de medio heterogéneo sobre un fondo impermeable de topografía irregular, con flujo horizontal en régimen transitorio; cuyas fronteras que limitan el dominio de flujo en todas direcciones se consideran como fronteras de flujo nulo, con excepción de una sección de la frontera sur, la cual se consideró con un nivel freático constante. El AG se alimenta por los escurrimientos del frente de montaña y por la recarga que generan las

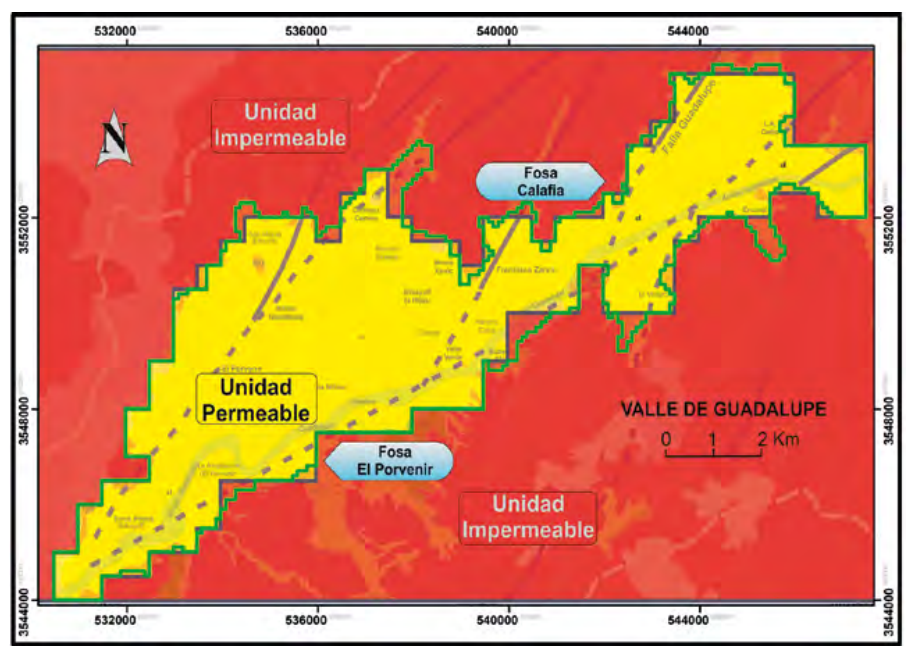

Figura 5. Geología del Valle de Guadalupe y dominio del flujo de agua subterránea (línea en azul obscuro). La línea verde representa la zona activa del modelo considerado en esta simulación. Imagen realizada con información de Campos-Gaytán et al. (2014).

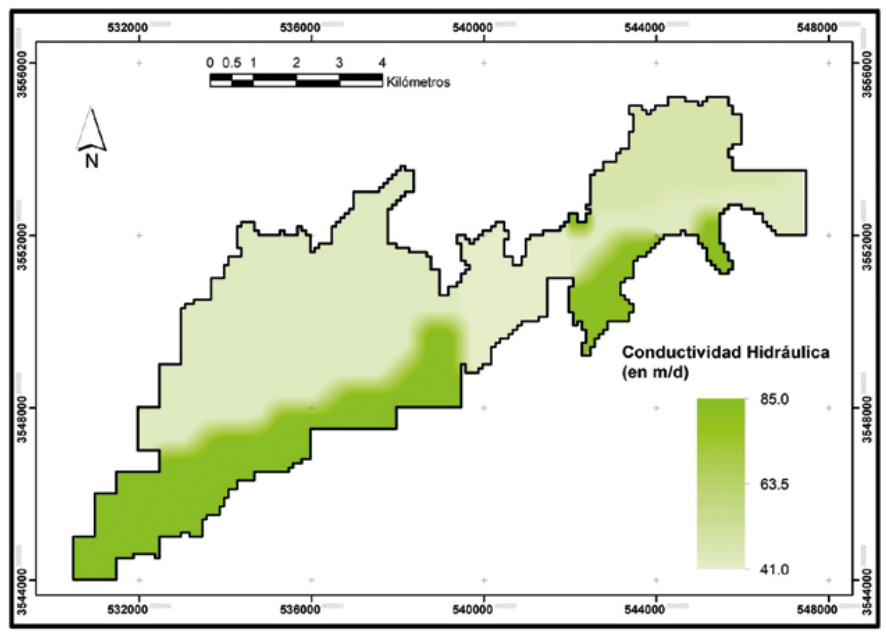

Figura 6. Conductividades hidráulicas (m/día) utilizadas en la simulación del flujo de agua subterránea en el AG.

Elaboración propia.

precipitaciones. Las descargas se componen del escurrimiento natural y las extracciones de los pozos que se ubican en la zona.

Dominio de flujo. La zona de estudio se discretizó en celdas cuadradas con longitudes de $100 \mathrm{~m}$ por cada lado, lo que generó una rejilla de 115 renglones, 180 columnas y una capa; se produjeron en total 20,700 celdas, de las cuales 5,600 se consideran activas (figura 7). En la misma figura se incluye la zona de drenes considerada durante el periodo completo de simulación, coincidente con el trazo del arroyo Guadalupe que cruza el acuífero. 


\section{INVESTIGAGIÓก Y CIECEIA DE LA UNIVERSIDAD AUTÓNOMA DE RGUASCALIERTES}

distribución de la recarga se muestra en la figura 8, y en ella se indica el porcentaje de recarga por el frente de montaña que corresponde a cada zona. Por la zona (h) se recarga $100 \%$ del escurrimiento superficial proveniente de la Ojos Negros.

El primer factor considerado como descarga de agua subterránea durante los estados estacionario y transitorio es la evapotranspiración ocurrida en el AG; para eso se han tomado en cuenta los valores de $60 \mathrm{~mm} / a n ̃ o$, para las zonas donde predomina la vegetación que alcanza en promedio los $3 \mathrm{~m}$ de altura (árboles de olivo en su mayoría) y $240 \mathrm{~mm} /$ año para zonas donde la vegetación predominante es de mucha menor altura (vid, forrajes, hortalizas y alfalfa), determinados por Campos-Gaytán (2008) (figura 8). agua subterránea en el AG, elaborada con información del MDE y publicada por el INEGI (s. f.). Trazo del dren incluido en el modelo.

Elaboración propia.

Elevación del terreno natural. Para definir la topografía superficial (figura 7) se utilizó la información publicada por el INEGI (s. f.) a través del MDE extraído del Continuo de Elevaciones Mexicano 3.0 (CEM 3.0), específicamente del área del estado de Baja California. Los espesores del acuífero en la fosa El Porvenir varían entre 15 y 100 m, mientras que en la fosa Calafia entre 50 y $255 \mathrm{~m}$. Estas profundidades están delimitadas por el contacto de la capa acuífera con la roca ígnea situada en el fondo, la cual se considera impermeable $\left(3.7 \times 10^{-7} \mathrm{a}\right.$ $3.7 \times 10^{-3} \mathrm{~m} / a n ̃ o$ ) (Campos-Gaytán, 2008). La anterior condición también es la que delimita las fronteras de flujo nulo laterales en todas las direcciones.

Recarga y descarga. La recarga de agua subterránea del AG se compone por las precipitaciones presentadas en toda la subcuenca Guadalupe y por los escurrimientos superficiales de la subcuenca Ojos Negros. Durante la simulación se han considerado: 1) La recarga por infiltración en la zona activa del modelo como un porcentaje de la precipitación directa en el acuífero (10\%), y otro porcentaje para la recarga por el frente de montaña (7\%). Este último se refiere a la precipitación presentada en el resto de la subcuenca Guadalupe y se considera como recarga en celdas perimetrales del modelo. 2) El volumen de agua registrado como escurrimiento superficial en la estación Agua Caliente, proveniente de la subcuenca Ojos Negros, se incluyó de manera directa como recarga del AG en las celdas ubicadas en el margen noreste. La

El segundo de los factores considerados para la descarga del modelo son los pozos de extracción y norias ubicadas dentro de la zona acuífera. Estas obras representan un volumen de extracción promedio de $28 \mathrm{Mm}^{3} / a n ̃ o$, utilizado principalmente para uso agrícola ( 20 $\left.\mathrm{Mm}^{3} / a n ̃ o\right)$ y el resto para suministro a la ciudad de Ensenada.

Mediciones del nivel freático en el AG. Durante el periodo comprendido desde 1978 hasta 1983 se registraron lluvias superiores al promedio anual en esta región. La recarga del AG por la infiltración

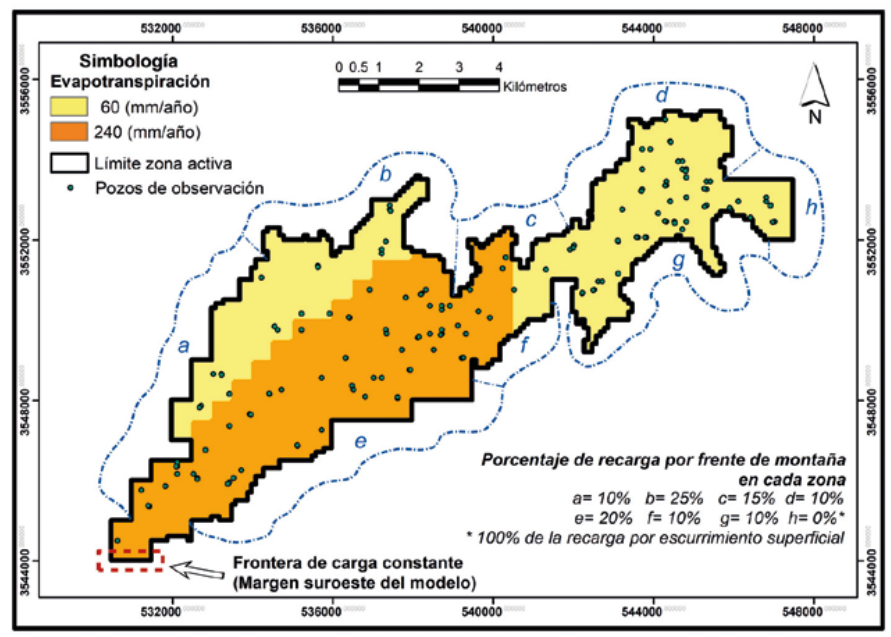

Figura 8. Distribución de la recarga por el frente de montaña considerada durante la simulación del agua subterránea en el AG. Tasa de pérdidas por evapotranspiración y ubicación de pozos de observación considerados para calibrar el modelo de agua subterránea en el AG. Localización de la frontera de nivel freático constante en el margen suroeste del modelo.

Elaboración propia. 


\section{IIVESTIGAGIÓn Y CUERCIA DE LA UกIVERSIDAD AUTÓOOOMA DE AGUASCALIERTES}

producto de dicho periodo continuó hasta febrero de 1984, fecha en que se registraron los niveles más altos en pozos monitoreados por la CONAGUA (CamposGaytán, 2008). Los niveles freáticos observados en el AG en febrero de 1984 se tomaron como referencia para evaluar la condición estacionaria. Para esto se consideró el registro de 35 pozos ubicados dentro del acuífero (figura 8).

Debido a que a lo largo de 30 años (1977-1998) se observó que en las celdas ubicadas en la salida del AG el nivel freático se ha mantenido constante con una variación de menos de 2 m (Campos-Gaytán, 2008), el margen suroeste se ha considerado como una frontera de carga constante (figura 8). Para la calibración de la condición transitoria se utilizaron los registros de 105 pozos de observación (figura 8).

\section{RESULTADOS}

Se realizó la simulación del flujo de agua subterránea en el $A G$ en los estados estacionario y transitorio. Los niveles freáticos simulados para la condición estacionaria se compararon con las elevaciones observadas en febrero de 1984, que varían entre los 285 m.s.n.m. al suroeste del dominio de flujo y los 362 m.s.n.m. al noreste. De la comparación de niveles freáticos calculados contra los observados se concluye que el ajuste vertical presentado es aceptable, y que el comportamiento del flujo de agua subterránea se logra reproducir satisfactoriamente con este modelo propuesto (figura 9). En cuanto a la condición transitoria los resultados del modelo de simulación para noviembre de 2008 se muestran en la figura 10 y se considera que, al igual que en la condición estacionaria, se logra reproducir de manera satisfactoria el comportamiento hidráulico observado en el acuífero.

En la figura 11 (a) se muestra la relación entre los niveles freáticos observado y simulado por el modelo, donde la mayoría coinciden con o se encuentran muy próximos a la línea de $45^{\circ}$. En la figura 11 (b) se muestran los volúmenes resultantes de la simulación del flujo de agua subterránea en

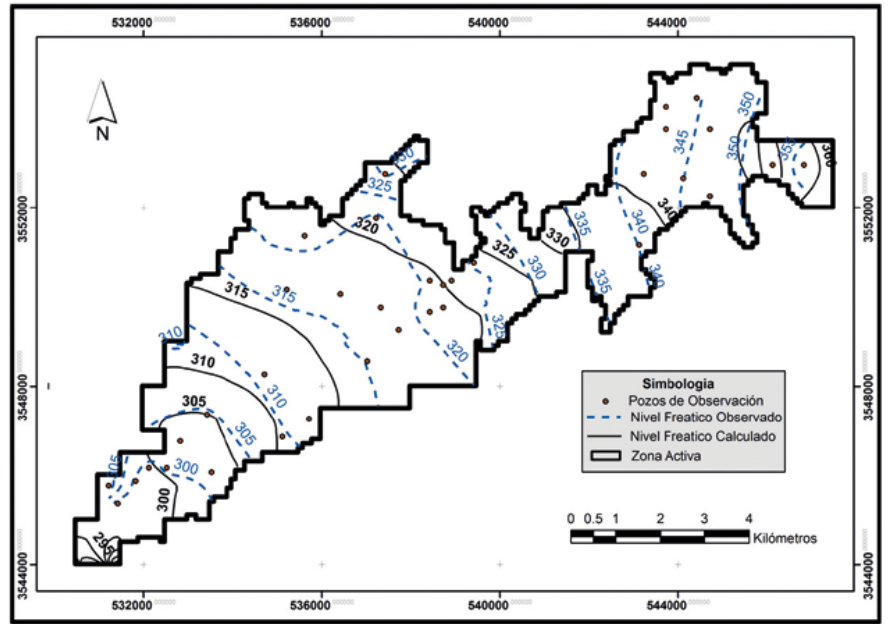

Figura 9. Niveles freáticos observado y simulado correspondientes a la fecha fijada como estado estacionario (febrero de 1984). Elaboración propia.

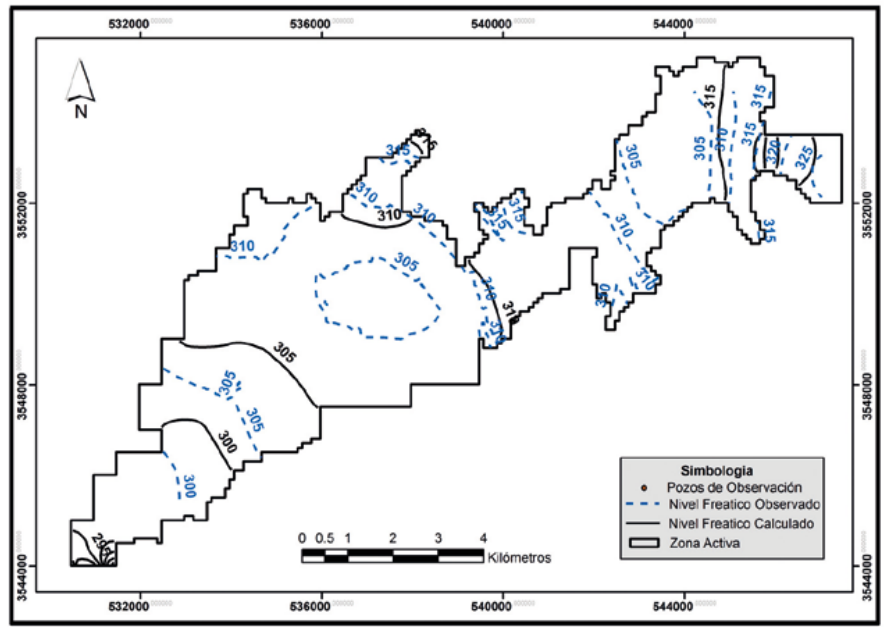

Figura 10. Niveles freáticos calculado y observado correspondientes a noviembre de 2008 en condiciones transitorias. Elaboración propia.

el AG en los estados estacionario y transitorio. Se puede observar que un volumen considerable de agua sale del acuífero por los pozos incluidos en el modelo, de una magnitud similar al que ingresa (recarga) al acuífero. 


\section{LA UחIVERSIDAD AUTÓnOMA}

DE RGUASCALIERTES

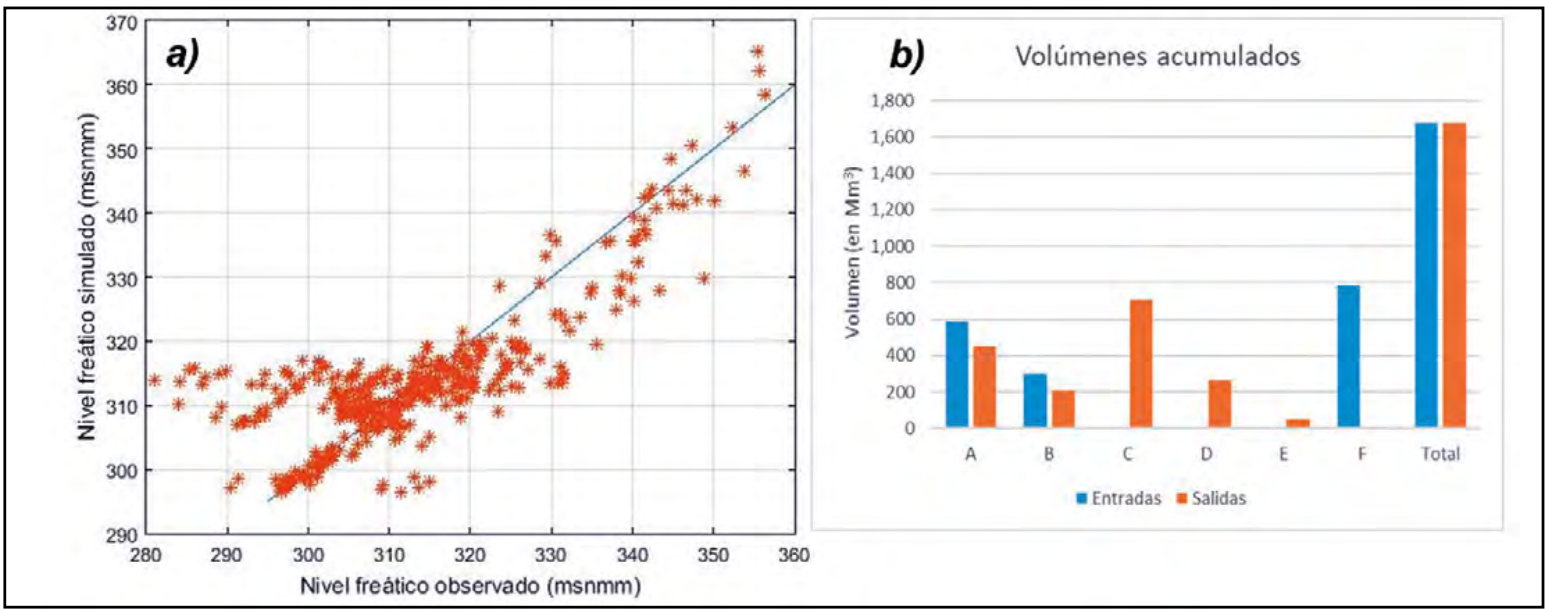

Figura 11. a) Diagrama de dispersión de la elevación de los niveles freáticos observado vs. simulado para la condición transitoria. b) Volúmenes totales para las variables que integran la ecuación de balance durante la simulación del agua subterránea en el AG en estado transitorio a diciembre de 2008 (A= Almacenamiento, B= Carga constante, $\mathrm{C}=$ Pozos, $\mathrm{D}=$ Drenes, E= Evapotranspiración, $\mathrm{F}=$ Recarga).

Elaboración propia.

Tabla 1

Estadísticos de resultados, observado vs. simulado

\begin{tabular}{cccc}
\hline Condición de simulación & RMSE $(\mathrm{m})$ & Residual Máximo $(\mathrm{m})$ & Residual Mínimo $(\mathrm{m})$ \\
\hline Estado estacionario & 3.25 & +14.40 & -0.190 \\
Estado transitorio & 8.36 & -32.94 & -0.003 \\
\hline
\end{tabular}

Nota: Elaboración propia.

Para el análisis de los resultados se utilizó el error cuadrático medio (RMSE, por sus siglas en inglés) como estadístico para comparar el ajuste que presenta el modelo. En la tabla 1 se muestra el RMSE y los residuales máximo y mínimo obtenidos (diferencia entre los niveles freáticos observado y simulado).

\section{DISCUSIÓN}

En los resultados se observa que la magnitud de agua que sale del acuífero por los drenes en condiciones estacionarias es cuantiosa $174 \%$ de las salidas del modelo), lo cual podría obedecer a que el acuífero en febrero de 1984 se encontraba en su capacidad máxima, debido a las lluvias extraordinarias presentadas en años inmediatos anteriores, por lo que el agua alcanza la elevación a la que los mismos entran en operación.

Por otra parte, el volumen de recarga generado por los escurrimientos superficiales provenientes de la subcuenca Ojos Negros representa $40 \%$ de la recarga total del $A G$, por lo que resultaría conve- niente la construcción de obras que permitan la retención de tales escurrimientos en la zona del acuífero para la recarga del mismo, lo que evita que este volumen se desaproveche si continúa su recorrido libre hasta su desembocadura en el océano.

Un modelo generado mediante MODFLOW-2005 (Harbaugh, 2005), software tan popular entre los investigadores en la actualidad, permite contar con una herramienta que puede ser compatible con programas de computadora especializados en otro tipo de problemática, por ejemplo, SUTRA (Voss \& Provost, 2010), utilizado para transporte de solutos y energía, o GWM (Ahlfeld et al., 2009), empleado para la optimización del recurso hídrico en acuíferos. Además, el MODFLOW-2005 (Harbaugh, 2005), mediante la interfaz ModelMuse (Winston, 2009), permite la inclusión de información de tipo ShapeFile, formato mediante el cual se incluyó el MDE.

En comparación con lo publicado por CamposGaytán et al. (2014), la condición estacionaria en cuanto al RMSE mejoró de $5.70 \mathrm{~m}$ a $3.25 \mathrm{~m}$ (>2.0 m de diferencia), como consecuencia de las modificacio- 


\section{IIVESTIGACIÓn Y CIEREIA DE LA UחIVERSIDAD AUTÓNOMA DE RGUASCHLIETTES}

nes realizadas con respecto al porcentaje de agua que se infiltra por el frente de montaña, tamaño de las zonas de recarga, mayor resolución en el número de celdas y reubicación del trazo de las celdas con dren (georreferenciado sobre el arroyo Guadalupe). En cuanto a la recarga por frente de montaña se modificó no solamente el porcentaje de 5\% utilizado en el modelo de Campos-Gaytán (2008) a 7\% para el actual modelo, además se consideró que este porcentaje se refiere a la precipitación que se presenta únicamente en el resto de la subcuenca Guadalupe, no al resto de la cuenca Guadalupe (subcuencas Ojos Negros-Real del Castillo). La georreferenciación del modelo también representa una ventaja, tanto para el manejo de la información como para su futura inclusión; es decir, si se requiere incluir pozos de observación, extracción o de otro tipo, se puede realizar con las coordenadas tomadas directamente en campo. Por otra parte, en la condición transitoria se alcanzó un valor para el RMSE de $8.36 \mathrm{~m}$ (evaluando el periodo 1984-2008) en comparación con los 7.1 m establecidos previamente por Campos-Gaytán et al. (2014) al evaluar el periodo 1984-2005.

En el diagrama de dispersión algunos puntos muestran un desfase con respecto a la línea de $45^{\circ}$, lo que podría obedecer a una situación muy específica referente a diferencias en las cotas de nivel topográfico de pozos de observación que presentan dos fuentes de información utilizadas para el diseño de este modelo. Por ejemplo, consideremos el caso del pozo de observación denominado VG2052, ubicado al noreste del modelo, cuya cota publicada por Campos-Gaytán (2008) indica que el brocal se encuentra a 355 m.s.n.m. y georreferenciándolo en el modelo aquí presentado se encuentra a 327 m.s.n.m. (INEGI, s. f.). Lo anterior podría generar errores en el ajuste del modelo, ya que esta información se toma en cuenta para establecer el nivel freático observado para determinada fecha.

Otro motivo que podría generar diferencia entre lo simulado por el modelo y lo observado en campo es un error en la medición al momento de capturar o procesar la información, no solo en relación con el ajuste del modelo. Debido a lo anterior sería recomendable realizar una revisión exhaustiva a las zonas que presenten mayor diferencia de nivel topográfico entre lo publicado por el INEGI y lo realizado físicamente por las dependencias locales.

Algo más a destacar en comparación con lo publicado por Campos-Gaytán et al. (2014) acer- ca de la obtención de un nivel freático subestimado para la fosa Calafia es que dicha situación se ha revertido en esta simulación. Esto puede obedecer a modificaciones hechas al modelo, como la nueva distribución de la recarga y el incremento en el volumen anual de recarga promedio (el cual pasó de 29 a $31 \mathrm{Mm}^{3}$ en este trabajo).

El modelo representa de manera precisa y satisfactoria el comportamiento del AG en los estados estacionario y transitorio, lo cual permite estimar parámetros como conductividad hidráulica, evapotranspiración y características de los drenes, apegadas a lo que sucede en campo y útiles para otros estudios en la región. Sin embargo, existen factores que limitan los alcances del modelo, entre ellos la falta de un registro constante del nivel freático. A pesar de que en fechas recientes se ha llevado a cabo un registro anual del nivel freático por parte de comités locales (COTAS del Valle de Guadalupe), la información histórica carece de constancia en cuanto a pozos monitoreados; es decir, en ocasiones las mediciones no se realizan en los mismos pozos y algunas lecturas tienen tres años de diferencia entre sí.

CONCLUSIONES

Se llevó a cabo la simulación numérica del flujo de agua subterránea en el AG, tanto en estado estacionario como transitorio, mediante el software MODFLOW-2005 (Harbaugh, 2005) y su interfaz ModelMuse (Winston, 2009), misma que reproduce satisfactoriamente la configuración del nivel freático observado desde febrero de 1984 hasta diciembre de 2008. Con respecto al comportamiento del modelo se concluye que: 1) la recarga que ingresa por las celdas al noreste de la rejilla, debido a los escurrimientos superficiales proveniente de las subcuencas Ojos Negros-Real del Castillo, representa la recarga de mayor magnitud para el modelo; 2) la descarga principal del modelo se realiza por drenes; 3) la modificación del criterio para establecer la recarga por el frente de montaña y la discretización del modelo a un número mayor de celdas (con tamaño de $100 \mathrm{~m} \times 100 \mathrm{~m}$ ) mejoraron el ajuste vertical entre los niveles freáticos simulado y observado en los pozos utilizados para la calibración del modelo en condición estacionaria. El ajuste obtenido en condición transitoria es similar al obtenido por Campos-Gaytán (2008), quien utilizó criterios de modelado (tamaño de la discretización y recarga) diferentes a los empleados en este trabajo. 
Contar con modelos de este tipo permite a las instituciones encargadas de gestionar los recursos hídricos conocer el comportamiento del flujo de agua subterránea en el acuífero, lo cual podría implementarse como criterio al momento de tomar decisiones que beneficien la disponibilidad de agua para las distintas actividades que del mismo dependen. Hoy día se cuenta con un modelo del AG que puede servir como base para estudiar e implementar una estrategia óptima de manejo del acuífero.

\section{REFERENCIAS}

- Ahlfeld, D. P., Baker, K. M., \& Barlow, P. M. (2009). GWM-2005-A Groundwater-Management Process for MODFLOW-2005 with Local Grid Refinement (LGR) capability, U.S. Geological Survey Techniques and Methods 6-A33. Reston, Virginia, US: US Department of the Interior-US Geological Survey.

- Camacho Garza, A. (2016). Análisis de las estrategias de adaptación a la escasez hídrica de las empresas vitivinícolas del Valle de Guadalupe, B. C. (Tesis de maestría). Recuperada de https://www.colef.mx/posgrado/wpcontent/uploads/2016/12/TESIS-Camacho-Garza-Abraham. pdf

- Campos-Gaytán, J. R. (2008). Simulación del flujo de agua subterránea en el acuífero del Valle de Guadalupe, Baja California, México (Tesis doctoral). Centro de Investigación Científica y de Educación Superior de Ensenada (CICESE), Ensenada, B. C., México.

\& Kretzschmar, T. (2006). Numerical understanding of regional scale water table behavior in the Guadalupe Valley aquifer, Baja California, Mexico. Hydrology and Earth System Sciences Discussions, 3(3), 707-730.

- Campos-Gaytán, J. R., Kretzschmar, T., \& Herrera-Oliva, C. S. (2014). Future groundwater extraction scenarios for an aquifer in a semiarid environment: Case study of Guadalupe Valley Aquifer, Baja California, Northwest Mexico. Environmental Monitoring and Assessment, 186(11), 7961-7985.

- Comisión Estatal del Agua de Baja California. (s. f.). Informes mensuales de diciembre de 2011 y de diciembre de 2016. Recuperados de http://www.cea.gob.mx/indicadores.html

- Comisión Nacional del Agua. (2015). Ubicación de aprovechamientos subterráneos y cuencas hidrográficas [Base de datos]. Recuperado de http://siga.conagua.gob. $\mathrm{mx} /$ REPDA/Menu/MenuKMZ.html

- Comisión Nacional para el Conocimiento y Uso de la Biodiversidad. (2004). Mapa base a nivel estatal. Formato vectorial [Mapa con ficha informativa]. Recuperado de http://www.conabio.gob.mx/informacion/metadata/gis/ mbaprgw.xml?_httpcache=yes\&_xsl=/db/metadata/xsl/ fgdc_html.xsl\&_indent=no

- Dentoni, M., Deidda, R., Paniconi, C., Qahman, K., \& Lecca, G. (2015). A simulation/optimization study to assess seawater intrusion management strategies for the Gaza Strip coastal aquifer (Palestine). Hydrogeology Journal, 23(2), 249-264.

- Harbaugh, A. W. (2005). MODFLOW-2005, The US Geological Survey modular ground-water model: The ground-water flow process. Techniques and Methods 6-A 19. Reston, VA, US: USGS.

- Herrera-Oliva, C. S., Campos-Gaytán, J. R., \& Carrillo-González, F. M. (2017). Estimación de datos faltantes de precipitación por el método de regresión lineal: Caso de estudio Cuenca Guadalupe, Baja California, México. Investigación y Ciencia de la Universidad Autónoma de Aguascalientes, 25 (71), 34-44.

- Instituto Nacional de Estadística y Geografía. (s. f.). Continuo de Elevaciones Mexicano 3.0 [Base de mapas descargables]. Recuperada de http://www.inegi.org.mx/geo/contenidos/ datosrelieve/continental/descarga.aspx

- König, C. M., Becker, M., Diehl, A., Seidel, T., Rosen, B., Rüber, O., \& Zimmermann, C. (2017). SPRING Simulation of Processesin Groundwater. User Manual. Witten, Alemania: delta-h Ingenieurgesellschaft mbH.

- Secretaría de Fomento Agropecuario de Baja California. (s. f.). Sistema de información para el manejo de agua de riego en Baja California [Base de datos interactiva]. Recuperado de http://www.simarbc.gob.mx/\#home

- Secretaría de Medio Ambiente y Recursos Naturales. (2013). Acuerdo por el que se actualiza la disponibilidad media anual de agua subterránea de los 653 acuíferos de los Estados Unidos Mexicanos, mismos que forman parte de las regiones hidrológico-administrativas que se indican. Diario Oficial de la Federación, 20 de diciembre de 2013.

- Villarreal Rodríguez, S. (2012). Variabilidad interanual de la evapotranspiración en dos ecosistemas semiáridos con patrones de precipitación distintos (Tesis de maestría). Centro de Investigación Científica y de Educación Superior de Ensenada (CICESE), Ensenada, B. C., México.

- Voss, C. I., \& Provost, A. M. (2010). SUTRA: A model for saturatedunsaturated variable-density ground-water flow with solute or energy transport. Reston, VA,US: US Geological Survey.

- Winston, R. B. (2009). ModelMuse-A graphical user interface for MODFLOW-2005 and PHAST. Reston, VA, US: US Geological Survey. 